\title{
Le HCR au Mexique auprès des Guatémaltèques : 20 ans de présence
}

\author{
Edith F. Kauffer Michel
}

\section{Résumé}

Alors que le Haut-commissariat des Nations Unies pour les réfugiés (HCR) est sur le point de fêter 20 ans de présence au Mexique, nous proposons une analyse du travail réalisé par cette institution dans ce pays en nous limitant plus particulièrement aux activités mises en ouvre auprès des réfugiés guatémaltèques.

Malgré les restrictions liées aux circonstances de l'arrivée du HCR au Mexique et à la position des autorités vis-à-vis du statut de réfugié et de la Convention de Genève, un des atouts qui a facilité son action a découlé de son rôle d'instance de financement. En conséquence, nous pouvons mentionner certaines actions en faveur des réfugiés guatémaltèques où l'intervention directe et indirecte du HCR a été fondamentale.

\section{Abstract}

As the United Nations High Commission for Refugees (UNHCR), prepares to celebrate twenty years of its presence in Mexico, this paper examines its work there, with special focus on the programs for Guatemalan refugees. Despite restrictions linked to the manner of its initial entry in Mexico as well as to the position adopted by the authorities towards refugees and the Geneva Convention, the UNHCR's activities were made easy because of its role as a source of financing. Consequently, we are able to mention some activities of the UNHCR in favour of Guatemalan refugees where its direct and/or indirect intervention proved crucial.

\section{Introduction}

TC mandat du HCR est défini par un statut qui a été adopté le 14 décembre 1950 par l'Assemblée générale des Nations Unies et qui détermine deux compétences particulières : la protection et l'assistance ${ }^{1}$. La première concerne les réfugiés dûment reconnus comme tels et se traduit par la recherche de solutions permanentes pour ces populations. Cette fonction implique des actions de concertation avec les autorités des pays d'installation, notamment lorsqu'il s'agit de les convaincre de recevoir les réfugiés. Parallèlement, le travail dirigé vers la mise en conformité des textes nationaux par rapport au droit international Conventionnel en matière de réfugiés dérive de la mission de protection du HCR. Cette activité de lobbying en faveur des réfugiés est conçue de manière à garantir une meilleure protection.

L'assistance est intimement liée à la protection, car elle consiste à satisfaire les besoins les plus pressants des réfugiés dans le pays d'accueil durant l'étape d'urgence. Et lorsque l'exil n'est pas temporaire, cette compétence inclut également la mise à la disposition des réfugiés de moyens qui facilitent leur intégration sur place.

" Entièrement apolitique, humanitaire et social ${ }^{2}$ » sont les adjectifs utilisés dans les textes pour désigner les activités réalisées par le HCR. La réalité est bien différente, car le thème des réfugiés est éminemment politique. Il s'agit d'un phénomène migratoire dont l'origine est liée à l'existence de crises politiques, de conflits armés, de dictatures militaires, et les réponses proposées par les différents États sont orientées en fonction de l'intérêt national.

Les activités développées par le HCR pour assurer la protection et l'assistance des populations réfugiées dépendent des décisions de son comité exécutif, instance formée par 
les États. Les politiques et les actions menées découlent ainsi de la disponibilité de donateurs qui apportent une aide selon les priorités du moment et selon les alliances entre États.

L'action du HCR auprès des réfugiés guatémaltèques au Mexique n'a évidemment pas échappé à ce schéma d'intérêts politiques des donateurs.

Une des difficultés majeures rencontrées sur le terrain était constituée par les limitations qui découlaient de la souveraineté mexicaine et de la non-reconnaissance des instruments du droit Conventionnel en la matière par ce pays récepteur. Comment le HCR a-t-il pu ainsi mener à bien ses fonctions auprès des Guatémaltèques? Comment l'institution a-t-elle pu dépasser les activités traditionnelles afin de constituer un appui tout particulier dans certains domaines?

Nous considérions que la clef de cette marge de manœuvre résidait dans son rôle d'agence de financement de la majorité des actions vis-à-vis des réfugiés guatémaltèques installés au Mexique, qui reposait sur une double stratégie. Finalement, le constat nous permet d'affirmer que le HCR a pu de cette manière mener à bien des actions exemplaires en faveur de la population bénéficiaire.

\section{Les restrictions à une action autonome}

L'action menée auprès des réfugiés guatémaltèques au Mexique s'inscrit dans un cadre restreint par la souveraineté de l'État mexicain; de ce fait, cette intervention est limitée. Après la description du contexte dans lequel se réalisaient les activités du HCR et leur évolution au cours des années, nous prendrons l'exemple de la réinstallation afin d'illustrer les limitations de son action.

\section{Le cadre juridique de la présence du HCR}

L'intervention du HCR au Mexique fut nécessaire au début de la décennie quatre-vingt lorsque le Mexique se vit dépassé par la magnitude des flux de réfugiés, notamment par l'arrivée de milliers de Guatémaltèques à sa frontière sud. Le 22 juillet 1980, un décret présidentiel instaura la Commission mexicaine d'aide aux réfugiés (COMAR) $)^{3}$. Le Mexique n'était pas signataire de la Convention de Genève, et le droit mexicain considérait uniquement le statut d'asile politique. Le 22 mars 1981, devant l'urgence de la situation fut signé un accord entre le HCR et le gouvernement mexicain qui définissait les bases de la coopération et le mécanisme de financement des projets. L'accord de siège date, quant à lui, du 5 octobre 1982 et fut approuvé par le Sénat le 17 décembre de la même année, puis publié dans le Journal officiel le 6 avril $1983^{4}$.

Le cadre juridique dans lequel s'inscrivait alors la présence du HCR au Mexique était extrêmement limité, et la non-souscription de cet État aux instruments internationaux ne facilitait guère la marge d'action auprès des réfugiés. Dans ce contexte, la souveraineté de l'État mexicain pouvait être utilisée à n'importe quel moment afin de limiter l'immixtion du HCR dans les affaires relatives aux réfugiés, et l'institution était en réalité sujette au bon vouloir des autorités mexicaines qui avaient la capacité de décider de sa présence sur son territoire. Cette volonté de limiter la marge de manœuvre du HCR n'était évidemment pas propre aux autorités mexicaines; elle peut être illustrée par d'autres exemples de pays récepteurs. Cependant, dans le cas qui nous intéresse, elle était d'autant plus tangible qu'elle s'ajoutait à l'inexistence du statut de réfugié et à la non-signature de la Convention de Genève par le Mexique.

Au cours des années, cette situation a évolué. La première amélioration se produisit en 1990 quand le Mexique approuva une modification à la loi générale de population et inclut le statut de réfugié dans le droit internes. Le règlement d'application de cette loi fut promulguéle 28 août $1992^{6}$, mais le document migratoire correspondant ne fut jamais distribué aux Guatémaltèques, ce qui a empêché cette réforme d'exercer un impact réel dans la vie quotidienne des réfugiés. Cependant, elle permit de signaler que les efforts menés par le HCR dans le domaine de l'adéquation de la législation nationale avaient porté leurs fruits.

La seconde mesure qui atténua la plus grande partie des restrictions juridiques à l'action du HCR au Mexique fut la récente signature de la Convention de Genève au début de l'année $2000^{7}$. Cette dernière permet une marge de manœuvre beaucoup plus large, même si la Convention fut signée avec un certain nombre de réserves. En réalité, cette décision intervint deux ans après la mise en marche de la politique d'intégration des réfugiés guatémaltèques, c'est-à-dire une fois qu'ils aient eu perdu leur statut de réfugié, et elle n'a donc eu aucun effet sur les actions menées par le HCR en leur faveur.

\section{Une marge d'action limitée : exemple de la réinstallation}

L'intervention du HCR auprès des réfugiés guatémaltèques au Mexique s'inscrivait dans un cadre juridique déterminé qui avait des effets sur les activités concrètes réalisées. L'épisode de la réinstallation dans les États du Campeche et du Quintana Roo illustra tout particulièrement cette réalité.

Afin de comprendre dans quelles circonstances se réalisa le transfert des réfugiés, nous rappellerons brièvement quelques faits.

L'arrivée de milliers de réfugiés entre 1980 et 1982 en territoire mexicain et leur installation spontanée dans l'État du Chiapas à faible distance de la frontière étaient dictées 
par leur ferme intention de rentrer au pays le plus rapidement possible. Leur présence gênait les autorités guatémaltèques qui, sous le prétexte de poursuivre de prétendus guerrilleros, franchirent la frontière et pénétrèrent à plusieurs reprises au Mexique. Cette violation de la souveraineté de son voisin s'accompagna d'actions violentes menées à l'encontre des Guatémaltèques dont certains citoyens mexicains furent malencontreusement les victimes. En avril 1984, les réfugiés du camp du Chupadero, situé à proximité de la frontière, furent attaqués par l'armée guatémaltèque, et sept d'entre eux furent assassinés. Devant cette tragédie, les autorités mexicaines décidèrent de manière unilatérale la réinstallation de la totalité des réfugiés présents au Chiapas vers les États du Campeche et du Quintana Roo situés dans la péninsule du Yucatán.

Cette décision prise dans le contexte des violations à la souveraineté mexicaine répondait aussi à la volonté de protéger la population réfugiée et mexicaine. La controverse de la réinstallation fut générée non pas par la décision en tant que telle, mais par les conditions dans lesquelles elle fut réalisée par les autorités lorsqu'elles se rendirent compte du refus des réfugiés. En effet, de nombreux Guatémaltèques s'opposèrent au transfert vers des terres inconnues et éloignées de leur pays d'origine.

Afin de surmonter leurs réticences, les autorités mexicaines utilisèrent diverses stratégies de persuasion accompagnées de méthodes coercitives. Ces dernières ne se limitèrent pas aux menaces de renvoi au Guatemala et à la suppression de l'aide humanitaire. Certaines furent appliquées notamment dans la zone la plus éloignée et la moins accessible qui porte le nom de Marqués de Comillas : la principale voie d'accès à cette région, la voie aérienne, fut fermée et surveillée afin de pouvoir mener à bien le transfert. Parmi les moyens employés pour convaincre les réfugiés se trouvait toute une gamme de techniques : promesse, persuasion, suppression de l'aide alimentaire et médicale allant jusqu'à l'incendie du camp de Puerto Rico. Les institutions qui participèrent aux opérations de transfert furent la Marine, les autorités migratoires et la COMAR. Le HCR fut exclu des opérations, et les membres de certaines organisations non gouvernementales (ONG) reçurent des menaces.

Quelle fut la réaction officielle du HCR? Publiquement, le HCR reconnut la nécessité de réaliser l'opération de réinstallation et le bien-fondé de cette décision. Quant aux moyens utilisés, les fonctionnaires s'abstinrent de faire des déclarations à ce sujet.

Cet épisode illustre parfaitement les limitations de l'action du HCR au Mexique et le caractère conditionné de son intervention. Devant la réinstallation, les fonctionnaires de l'institution optèrent pour le silence afin d'assurer la continuité de leur présence au Mexique.

Au fil des années, la stabilisation de la situation des réfugiés, l'ouverture du Mexique à une politique plus humanitaire et les transformations législatives permirent au HCR d'élargir sa marge de manœuvre.

\section{L'avantage du financement}

Dans de nombreux pays où le HCR intervient, il agit comme une agence de financement qui canalise les donations à travers des instances nationales d'attention aux réfugiés, afin de mener à bien les actions de protection et d'assistance. Son statu ${ }^{8}$ permet clairement la distribution de fonds à des organismes publics ou privés nationaux. Dans le cas du Mexique, il est possible d'évoquer une stratégie de double financement qui fut utilisée pour faire admettre certaines lignes d'action ou développer des projets particuliers.

\section{La stratégie du double financement: officiel et non gouvernemental}

Pour comprendre pourquoi le fait de financer à la fois des agences gouvernementales et des organisations non gouvernementales peut s'interpréter comme une stratégie, il est nécessaire de contextualiser l'intervention du HCR au Mexique vis-à-vis des institutions gouvernementales et des ONG.

À l'arrivée des Guatémaltèques et jusqu'en 1989 prédomina dans les sphères officielles une vision du problème orientée vers la sécurité nationale. En conséquence, l'action des ONG auprès des réfugiés fut restreinte d'une manière tout à fait consciente par les autorités. Plusieurs ONG parvinrent cependant à travailler de manière discrète durant plusieurs années.

C'est à partir de cette expérience que commença à s'observer une opposition entre l'action menée par les ONG et les activités réalisées par les autorités. Les premières considéraient œuvrer en faveur de la population réfugiée et affirmaient que les secondes agissaient seulement dans le but de contrôler les Guatémaltèques. Les secondes accusaient les premières d'utiliser les réfugiés comme un prétexte pour alimenter leur opposition au gouvernement en place ou pour obtenir un financement international. Cette fracture historique n'a pas pu être dépassée, même lorsque la perception du gouvernement s'orienta vers une vision davantage humanitaire et abandonna la logique sécuritaire.

Dans ce contexte, après avoir financé exclusivement la COMAR jusqu'en 1989, le HCR commença à appuyer les activités des ONG, dont la présence fut alors acceptée officiellement par les autorités. 


\section{Le financement ou la possibilité d'imposer certaines lignes d'action}

La majeure partie du financement existant pour développer des actions auprès des réfugiés guatémaltèques transitait à travers le $\mathrm{HCR}^{9}$, et d'une certaine manière ce rôle d'agence financière lui octroyait la possibilité d'orienter son apport économique vers des projets définis, à la fois à travers les institutions gouvernementales et les oNG.

Par exemple, au cours des années d'activité intense auprès des réfugiés guatémaltèques, la majorité des postes de la COMAR, agence interministérielle mexicaine, étaient directement financés par le HCR, alors que certains fonctionnaires rémunérés par le gouvernement mexicain recevaient une indemnité versée par le HCR. Cette situation permit bien évidemment au HCR d'agir dans des conditions plus favorables, car le salaire du personnel mexicain dépendait de la présence de l'institution internationale.

C'est surtout à travers le financement des activités des ONG que le HCR parvint à imposer clairement ses priorités, car les projets proposés étaient réalisés de manière conjointe. Le fait que la survie des ong dépendait de la continuité du financement apporté par le HCR définissait les termes de la négociation des projets : un projet qui n'intéressait pas le HCR avait de faibles chances d'être approuvé. Quant aux lignes prioritaires d'action du HCR, elles finissaient généralement par déboucher sur certains projets menés à bien par des ONG locales.

Même si la majorité des activités développées par la COMAR auprès des réfugiés dépendaient économiquement du HCR, le financement ne garantissait nullement une prise en compte totale des priorités de celui-ci par les autorités mexicaines, car les limitations déjà évoquées étaient réelles. Cependant, les activités des ONG représentaient un contrepoids : les actions que le HCR ne pouvait pas développer à travers le canal gouvernemental se réalisaient grâce au financement accordé aux ONG. C'est dans ce sens que nous considérons qu'un des principaux atouts de l'exercice diplomatique compliqué mené par le HCR au Mexique a consisté en une double stratégie de financement qui permit à la fois de concilier l'exigence de respect de la souveraineté mexicaine et de développer des actions qui allèrent bien au-delà de la protection et de l'assistance.

\section{Des exemples d'actions exemplaires impulsées et soutenues par le HCR}

En réalité, malgré les restrictions mentionnées, nous pouvons évoquer certaines actions en faveur des réfugiés qui ont été appuyées de manière décidée par le HCR au cours des années d'exil au Mexique. Nous choisirons de décrire trois contributions majeures, dont deux d'entre elles s'inscrivent dans le cadre des solutions apportées au refuge : le retour et l'intégration. Quant à la troisième, elle concerne le rôle tout particulier apporté aux activités des femmes, principalement en matière d'organisation.

\section{Le retour: coopération politique et financière du HCR}

La mobilisation politique autour du retour et l'organisation de cette expérience inédite ont été tout particulièrement soutenues par le HCR sur le plan politique aussi bien à l'échelle locale qu'internationale, et le retour a bénéficié d'un financement spécial. Avant d'analyser ce double apport du HCR dans le processus de retour au Guatemala, nous tenterons d'apporter quelques éclaircissements relatifs au terme de retour et à l'histoire de cette expérience.

Le retour constituait l'une des deux modalités de rentrée au pays offertes aux réfugiés guatémaltèques et il s'opposait en cela au rapatriement individuel. Les premiers rapatriements individuels commencèrent dès 1984, et les rapatriés étaient alors inclus dans le schéma de reconstruction militarisée du Guatemala. En 1987, à la suite de la signature d'un accord, entre le Mexique, le Guatemala et le HCR, sur le thème du rapatriement qui déboucha sur la création d'une instance spéciale chargée des populations rapatriées et déplacées appelée Commission spéciale d'attention aux rapatriés et déplacés (CEAR), le président guatémaltèque Cerezo fit campagne dans les camps de réfugiés pour promouvoir le rapatriement.

En réponse à cette possibilité qui n'était pas entièrement satisfaisante pour eux, certains réfugiés s'organisèrent pour former les Commissions permanentes des représentants des réfugiés guatémaltèques au Mexique (CCPP), qui, dès lors, entreprirent de définir les conditions dans lesquelles devait s'effectuer le retour au Guatemala. Le 8 octobre 1992 furent signés les accords sur le retour avec les autorités guatémaltèques $^{10}$, et le 20 janvier 1993 se réalisa le premier retour.

La principale différence entre le retour et le rapatriement résidait dans le caractère collectif, organisé du premier à la différence du second, individuel ou familial. De même, le retour impliquait certaines garanties et conditions définies par les réfugiés, tandis que le rapatriement ne permettait pas la participation de la population dans la détermination de conditions. Enfin, alors que le rapatriement signifiait la rentrée physique des réfugiés, le retour s'articulait à un projet politique de transformation et de démocratisation.

Le soutien apporté par le HCR au retour au Guatemala peut se classifier en deux domaines : politique et économique. 
Sur le plan politique et à l'échelle locale, le HCR a reconnu, dès leur formation, la légitimité des CCPP et leur caractère d'interlocuteur. De même, bien que participant dans l'organisation des rapatriements, le HCR a sans cesse soutenu la volonté des réfugiés qui désiraient rentrer sous la modalité du retour.

La signature des accords entre les CCPP et le gouvernement guatémaltèque se produisit à la suite d'un long processus de négociations au cours duquel les positions des deux parties semblaient inconciliables. Les réfugiés exigeaient certaines conditions que les autorités refusaient obstinément de considérer. Finalement, le contexte international et la mauvaise image liée à la présence de milliers de réfugiés hors du territoire guatémaltèque furent décisifs. De fait, le HCR intervint directement et participa à la pression internationale et au plus haut niveau : en novembre 1991, la haut-commissaire visita les camps de réfugiés du Chiapas avant de se rendre au Guatemala, acte qui signifiait un soutien inconditionnel du HCR à la cause du retour.

Bien entendu, le HCR ne fut pas la seule instance qui encouragea le projet politique de retour : les autorités mexicaines ainsi que les ONG locales, nationales et internationales participèrent à cet effort.

Sur le plan économique, le financement du retour peut se diviser en deux types d'actions, les indirectes et les directes.

Le HCR fut la principale source de financement des activités de formation de la population réfugiée réalisées dans le contexte du retour avec l'aide des ong. Cet apport permettait d'alimenter de manière indirecte l'organisation de la population décidée à retourner au Guatemala, grâce aux activités de formation qui assurèrent un apprentissage et une prise de conscience parmi les réfugiés.

L'apport économique direct consistait, quant à lui, en un financement de la logistique des retours : les moyens de transport des personnes et des biens, les services de santé, la nourriture, tout le nécessaire pour que la rentrée au pays s'effectue dans des conditions optimales.

À la différence des organisations de femmes impulsées par le HCR, le retour surgit directement des réfugiés. Cependant, le soutien multiforme apporté par le HCR fut décisif dans sa réalisation.

\section{L'intégration locale : ultime contribution du HCR}

L'intégration locale figure parmi les trois solutions préconisées pour mettre fin aux situations de réfugiés, les deux autres étant le rapatriement et la réinstallation dans un pays tiers. Cependant, il s'agit d'une solution guère mise en ouvre par les pays récepteurs qui considèrent que le rapatriement vers le pays d'origine est celle la plus indiquée. En conséquence, il existe peu d'exemples d'intégration locale de réfugiés à travers le monde. Depuis $1996 \mathrm{au}$ Campeche et au Quintana Roo et à partir de $1998 \mathrm{au}$ Chiapas, le gouvernement mexicain a ouvert cette possibilité aux Guatémaltèques. À la différence du retour et à l'exclusion d'un petit groupe qui réclamait la possibilité de rester au Mexique depuis 1993, l'intégration locale s'imposa comme une décision de politique étrangère largement suggérée par le HCR et financée en partie par l'institution.

Dans la genèse de la politique d'intégration, le rôle du HCR consista à convaincre les autorités guatémaltèques et mexicaines que l'intégration constituait le complément idéal du retour et du rapatriement. Pourtant, il devint évident que ces deux processus étaient en train de perdre, pour des raisons diverses, leur caractère attractif et que la présence des Guatémaltèques en territoire mexicain avait créé les conditions d'une résidence définitive et permis de tisser des liens affectifs difficilement dissolubles.

L'acceptation des autorités mexicaines fut favorisée par la sensibilité de certains fonctionnaires, par l'existence d'une ancienne proposition similaire qui n'avait pu, en raison des circonstances de mobilisation en faveur du retour, être menée à bien et par les conditions générales du refuge qui indiquaient une intégration de facto.

Bien que ne représentant plus la source principale de financement dans ce nouveau contexte, car l'Union européenne qui canalisait dans le passé les fonds apportés à travers le HCR décida de créer un bureau spécialisé en collaboration avec le gouvernement mexicain, le HCR participa grâce au financement de certains services et infrastructures afin de se séparer définitivement des personnes devenues alors des résidents dans des conditions optimales.

\section{L'organisation des femmes : impulsion et soutien inconditionnel du HCR}

En contraste avec l'image traditionnelle du réfugié victime, mourant de faim et dépendant de l'aide internationale, l'expérience des réfugiés guatémaltèques au Mexique nous présente un panorama de vitalité organisationnelle et de mobilisation politique sans précédent qui aboutirent au retour collectif et organisé de milliers de personnes dans des conditions définies par elles-mêmes et que les autorités guatémaltèques durent accepter contre leur volontén ${ }^{11}$

C'est dans ce contexte, favorisé par le HCR, qu'apparurent les organisations de réfugiées guatémaltèques, et ce 
phénomène fut impulsé et soutenu de manière résolue par l'institution.

En effet, comment expliquer que les réfugiées guatémaltèques d'origine indienne, pour la majorité analphabètes et ne parlant pas espagnol, exclues traditionnellement de la sphère publique aient pu participer dans des organisations de femmes et, pour certaines, se transformer en véritables leaders de ces groupes organisés? Le HCR ne fut pas étranger à cette dynamique.

À l'échelle internationale et à la suite des terribles expériences vécues par les réfugiées du sud-est asiatique, les politiques du HCR commencèrent à incorporer une distinction fondamentale à partir des années quatre-vingt. Les responsables des programmes jusque-là conçus pour un bénéficiaire asexué estimèrent alors que l'expérience d'un réfugié et celle d'une réfugiée devaient être différenciées. Ils jugèrent qu'il était nécessaire de développer des actions de protection et d'assistance particulières dirigées vers la population féminine en prenant en compte non seulement les rôles traditionnels de mère, de fille et d'épouse, mais aussi leur participation dans les activités productives, administratives et organisationnelles.

Au Mexique, le contexte dans lequel se définit la condition de réfugié favorisa de manière indéniable le processus de participation et d'organisation des femmes. En effet, durant la période d'exil, la traditionnelle différenciation des fonctions sociales entre hommes et femmes eut tendance à se transformer notamment à l'égard de l'impossibilité pour les réfugiés du sexe masculin de continuer à assumer les fonctions inhérentes à leur masculinité, entendue comme socialement et culturellement définie. Nous faisons notamment allusion au rôle de père de famille, travailleur qui apporte les ressources économiques. Ce rôle fut difficile à perpétuer dans les conditions d'exil en raison de la situation migratoire des réfugiés, de l'irrégularité de l'emploi et du fait qu'il pouvait être substitué en partie par l'aide alimentaire.

La déterritorialisation inhérente à la condition de réfugié permit également une plus grande flexibilité des normes sociales et ouvrit l'option, pour les femmes, de participer à de nouvelles activités à l'extérieur de leurs foyers.

Dans ce contexte facilité par la situation vécue en exil et par la transformation des fonctions masculines et féminines, le surgissement de ces organisations des femmes réfugiées ne fut cependant pas l'effet du hasard. Le premier pas vers la formation d'organisations trouva son origine dans une initiative du personnel du HCR, qui entreprit de manière patiente de visiter les camps de réfugiés afin de tenter de convaincre un premier groupe de femmes. Devant leur réaction négative motivée par de multiples raisons, notamment le manque de confiance en soi, la crainte de provoquer la colère de l'époux, le désintérêt et la perte de temps, les personnes qui étaient convaincues du bien-fondé de cette idée ne lâchèrent pas prise et parvinrent à impulser de manière graduelle les organisations de femmes réfugiées.

La création de ces organisations, qui existèrent jusqu'à ce que la majeure partie de leurs membres retournèrent définitivement au Guatemala, fut stimulée par le HCR au fil des années. Cet encouragement fut alimenté par de nombreuses ONG qui travaillèrent auprès des femmes et aidèrent les organisations en émergence dans divers domaines : éducation, santé, artisanat, droits humains, formation en général.

Bien évidemment, cette expérience fut possible grâce à l'existence d'un financement canalisé par le HCR et destiné à soutenir l'activité des organisations de femmes réfugiées et des ONG.

Devant les résistances des époux et des dirigeants réfugiés, le HCR entreprit un processus de formation et de persuasion des hommes, accompagné d'un effort soutenu destiné à convaincre les institutions mexicaines de l'impératif de soutenir les organisations de femmes.

Afin que leur participation ne demeurât pas théorique et ne fût pas empêchée par les activités domestiques, le HCR et les ONG mirent à leur disposition un certain nombre de services, comme les garderies et un programme de distribution de gazinières qui prétendaient remplacer le rustique foyer alimenté au bois. L'ensemble des actions dirigées vers l'organisation des femmes réfugiées procédait en effet d'une vision intégrale.

Ces efforts aboutirent à la formation de trois organisations de femmes: Mama Maquín, Madre Tierra et Ixmucané. La première fut créée en 1990 et les deux autres, au cours des années suivantes. Mama Maquín sut regrouper un total de 9000 membres dans les trois États de réception et animer une série d'activités orientées vers la formation, la réflexion et la prise de conscience des femmes. L'impact des deux organisations restantes fut moindre, bien que fondamental pour leurs participantes. Les trois groupes s'inscrivirent dans le contexte du retour au Guatemala et abandonnèrent le Mexique pour s'installer dans le pays d'origine et continuer leur labeur auprès des femmes retournées.

\section{Conclusion}

La signature de la Convention de Genève au début de l'an 2000 permit de constater que, malgré les restrictions, le HCR a pu gagner un terrain considérable au Mexique, ce qui lui 
permettra de se retirer avec une certaine tranquillité, une fois la question des réfugiés guatémaltèques réglée grâce à leur intégration à la société d'accueil à travers l'obtention de la nationalité mexicaine. Les futurs demandeurs du statut de réfugié demeurent, quant à eux, protégés par l'adhésion du Mexique au droit Conventionnel international.

Quant aux «ex-réfugiés » guatémaltèques — ainsi se considèrent-ils - rentrés au Guatemala pour certains, mexicanisés ou sur le point de l'être pour d'autres, ils conserveront à tout jamais dans leur mémoire le souvenir de ces dizaines de personnes venues d'autres pays arborant un logotype bleu et blanc, dont la mission consista à les accompagner un instant sur un chemin incertain et à les aider à imaginer comment construire un avenir meilleur.

\section{Notes}

1. Alto Comisionado de Naciones Unidas para los Refugiados, «Estatuto de la oficina del Alto Comisionado de las Naciones Unidas para los Refugiados ", Compilación de instrumentos jurídicos internacionales: principios y criterios relativos a refugiados y derechos humanos (San José : ACNUR, 1992).

2. Ibid., 111 .

3. Diario Oficial de la Federación, Primera sección (Mexico : 22 juillet 1982).

4. Diario Oficial de la Federación (Mexico : 6 avril 1983).

5. Diario Oficial de la Federación, "Ley General de Población" (Mexico : 17 juillet 1990).

6. Diario Oficial de la Federación, « Reglamento de la Ley General de Población» (Mexico : 21 août 1992).

7. UNHCR Press Realeases, UNHCR Hails Mexico's Accession to International Refugee Intruments, (20 avril 200o), en ligne : $<\mathrm{http}: /$ /www.unhcr.ch/news/pr/prooo420.htm $>$ (date d'accès : avril 2001).

8. Alto Comisionado de Naciones Unidas para los Refugiados, 114.

9. Certaines ONG européennes réalisaient des contributions directes dans le cadre de projets particuliers.

10. Comisiones Permanentes de Representantes de Refugiados Guatemaltecos en México, Comisión Nacional para la Atención a Refugiados, Repatriados y Desplazados, El Gobierno de la República de guatemala representado por la CEAR y las CCPP (Guatemala : 8 octobre 1992).

11. Edith Kauffer, Les réfugiés guatémaltèques au Chiapas. Le retour du peuple du maïs. Un projet politique (París : L'Harmattan, 2000).

Politicologue, Edith F. Kauffer Michel est chercheuse au Colegio de la Frontera Sur, San Cristóbal de Las Casas, Chiapas, Mexique. Elle travaille actuellement sur la politique d'intégration des réfugiés guatémaltèques au Mexique.

(C) Edith F. Kauffer Michel, 2001. Cette œuvre en libre accès fait l'objet d'une licence Creative Commons Attribution-NonCommercial 4.0 International License, laquelle autorise l'utilisation, la reproduction et la distribution de l'œuvre sur tout support à des fins non commerciales, pourvu que l'auteur ou les auteurs originaux soient mentionnés et que la publication originale dans Refuge : revue canadienne sur les réfugiés soit citée. 